\title{
Profiling of NUMTs in Gingivobuccal Oral Cancer
}

\author{
Arindam Palodhi ${ }^{1}$, Tanisha Singla ${ }^{1}$, Arindam Maitra ${ }^{{ }^{*}}$
}

${ }^{1}$ National Institute of Biomedical Genomics, Kalyani, India

* To whom correspondence should be addressed (am1@nibmg.ac.in)

\section{Abstract}

Very little information exists on the NUclear MiTochondrial sequences (NUMTs) in oral squamous cell carcinoma-gingivobuccal (OSCC-GB). We analysed whole genome sequences obtained from paired tumour and blood DNA from 25 patients of OSCC-GB for detecting NUMTs. Nine potential somatic NUMTs and 78 germline NUMTs were identified in these patients. None of the somatic NUMTs could be confirmed by PCR assay. None of the germline NUMTs were found to be specific for OSCC-GB. Although there have been recent reports on detection of somatic NUMTs in other cancer types, our results suggest NUMTs, both somatic and germline, are not involved in OSCC-GB.

\section{Introduction}

The mtDNA fragments localized into the nuclear genome are identified as NUclear MiTochondrial sequences (NUMTS) and the processes which generate NUMTs are collectively known as NUMTogenesis (Singh et al., 2017). Irrespective of the insertion site and the mtDNA sequence, all NUMTs found in human are rendered non-coding. This pseudogenization of NUMTs is due to the difference in codon usage between the nDNA and the mIDNA (Gellissen et al., 1987; Perna et al., 1996).

Although role of NUMTs in mitochondrial-nuclear genome evolution has been studied considerably, information on the de-novo NUMTs in both sporadic (Turner et al., 2003) and inherited diseases (Goldin et al., 2004) are scarce. Involvement of NUMTs in cancer remains a relatively underexplored yet important area of research.

\section{Study participants and methods}

Twenty-five patients of gingivobuccal oral cancer (OSCC-GB) were recruited with voluntary informed consent (ICGC India Project Team, 2013). Blood samples collected with informed consent from seven non-cancer individuals were used as control samples.

Whole genome sequence data was generated from paired tumor and blood DNA of 25 OSCCGB patients by paired end sequencing using Illumina Hiseq-2500. BWA-MEM (Li, 2013) was used to align the whole genome FASTQ files to the human reference sequence (hg19) + mitochondrial reference sequence (rCRS- revised Cambridge Reference Sequence). Alignment files thus produced were used as input for Discovery of Nuclear Mitochondrial Insertions 
(DINUMT; Dayama et al., 2014) to identify NUMTs. Circos plots shown in this study were generated using BioCircos (Cui et al., 2016).

For the PCR validation of the NUMTs, the individual primer pairs were designed in a manner, such that they would amplify the entire length of the NUMT + at least 150 bases upstream + at least 150 bases downstream of the insertion site and in absence of NUMTs they will amplify only the buffer region (Supplementary Table S1, Supplementary Table S2).

\section{Results}

NUMTs detected in both paired tumours-blood samples were defined as germline NUMT (GNUMT) and NUMTs present only in tumours samples but not in paired blood DNA were defined as somatic NUMT (S-NUMTS). Using DINUMT, we could detect 50 such S-NUMTs in 25 OSCC-GB patients. Each of these S-NUMTs were visually inspected using IGV (Robinson et al, 2011). After verification in IGV, we found 9 potential S-NUMTs in 6 patients were supported by at least two discordant reads and one split read (Figure 1A, Table 1). Only these 9 S-NUMTS which were supported by both discordant and split reads were investigated further.
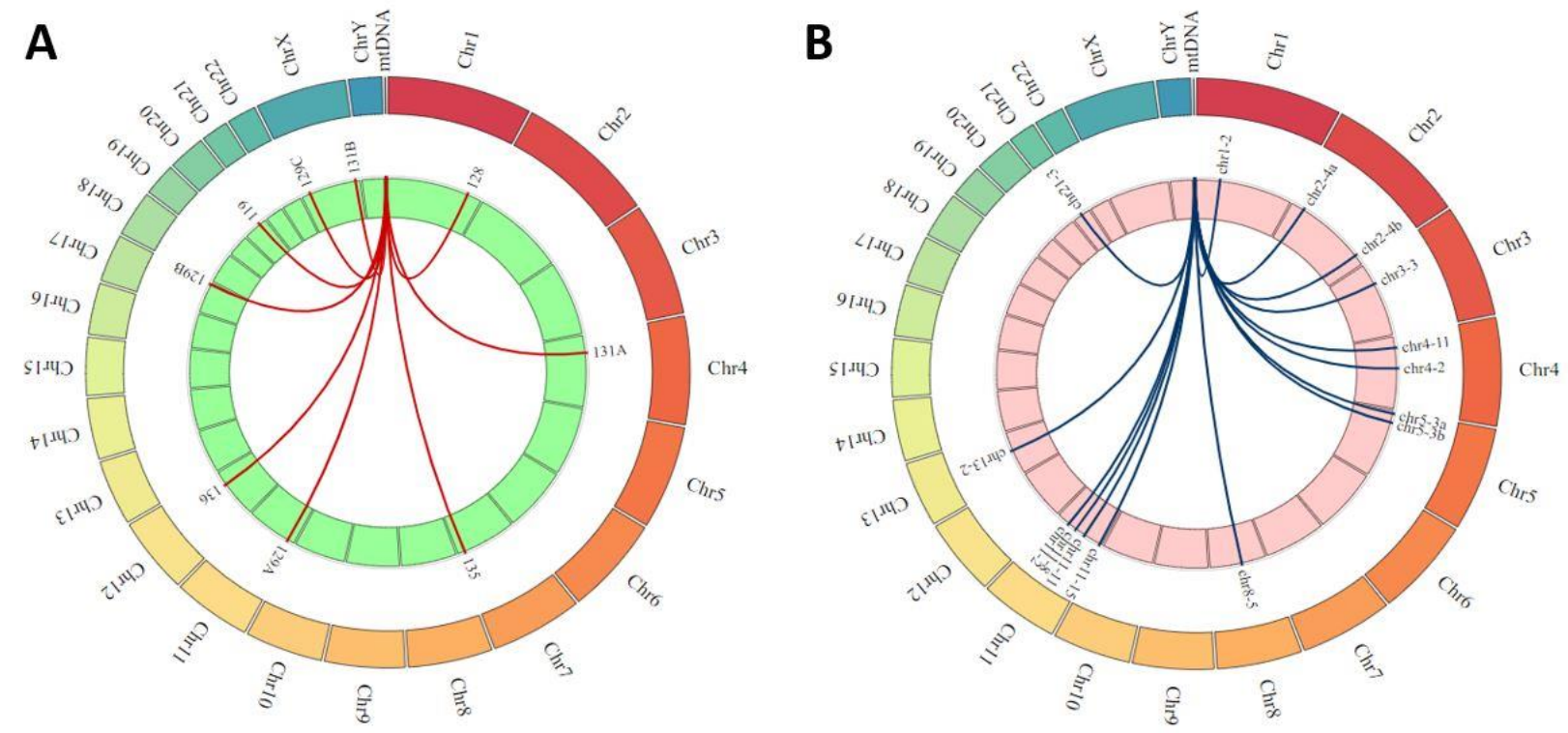

Figure 1: (A) Circos plot representing insertion location of S-NUMTs across chromosomes as identified by DINUMT. (B) Circos plot representing insertion location of G-NUMTs across chromosomes as identified by DINUMT.

\begin{tabular}{|ccccc|}
\hline Chr. No. & $\begin{array}{c}\text { Insertion } \\
\text { Start }\end{array}$ & $\begin{array}{c}\text { Insertion } \\
\text { Stop }\end{array}$ & $\begin{array}{c}\text { NUMT } \\
\text { length }\end{array}$ & Patient No. \\
20 & 45709144 & 45709146 & 3895 & 119 \\
1 & 210731831 & 210731888 & 6770 & 128 \\
11 & 17491095 & 17491097 & 5087 & $129 \mathrm{~A}^{*}$ \\
17 & 77335608 & 77335610 & 4304 & $129 \mathrm{~B}^{*}$ \\
$\mathrm{X}$ & 22480071 & 22480095 & 1933 & $129 \mathrm{C}^{*}$ \\
4 & 47023035 & 47023211 & 5436 & $131 \mathrm{~A}^{*}$ \\
$\mathrm{X}$ & 144335757 & 144335899 & 559 & $131 \mathrm{~B}^{*}$ \\
7 & 140921797 & 140921880 & 5958 & 135 \\
12 & 94944428 & 94944538 & 585 & 136 \\
\hline
\end{tabular}


Table 1: List of somatic mutations identified by DINUMT with their chromosomal location, length of the mitochondrial insert and patient no. *NUMTs were designated alphabetically when a single patient harbour more than one S-NUMTs.

A total of 78 G-NUMTs were identified in 25 patients (Figure 1B, Table 2). We named the GNUMTs as follows: chr-NO.-occurrence. Five recurrently reported G-NUMTs present in chromosome 11 (2nos; chr-11-15, chr-11-11) chromosome 8 (chr-8-5), chromosome 4 (chr-411) and chromosome 2 (chr-2-4) were selected for confirmatory assay. Additionally, no previously reported NUMTs were found in the neighbouring genomic region (1 $\mathrm{kb}$ upstream to $1 \mathrm{~kb}$ downstream of the predicted insertion site) from each of the identified G-NUMT sites by UCSC genome browser with the NUMT track.

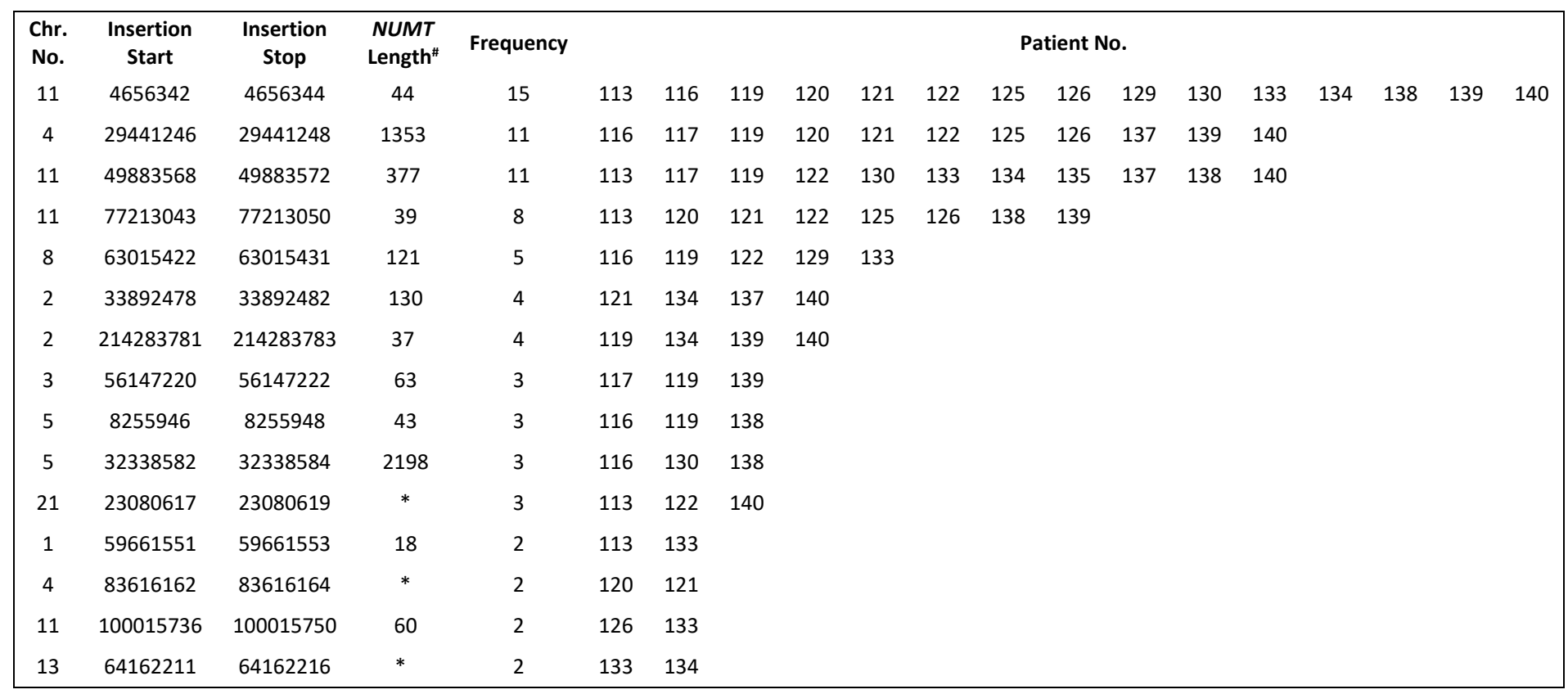

Table 2: List of germline mutations identified by DINUMT with their chromosomal location, length of the mitochondrial insert, frequency in the patients and the patients harbouring those NUMTs. " length of the longest NUMT observed in any of the patient carrying that NUMT is reported. *length of the NUMT could not be determined by DINUMT.

In $4(119,129 C, 131 B, 136)$ out of the 9 potential S-NUMTs, PCR-amplicons of length spanning the respective S-NUMT and the buffer region was observed from both tumour and matched blood. In two potential S-NUMTs $(128,131 \mathrm{~A})$, amplification of only buffer region was observed from both tumours and blood. No amplicons were observed for rest of the three potential S-NUMTs (129A, 129B, 135). 
G-NUMT chr2-4 was detected in all 4 patients by PCR. G-NUMT chr8-5 was found to be present in blood and tumour DNA in only one patient. Presence of G-NUMT chr4-11 was detected in 3 OSCC-GB patients. Only three patients carried G-NUMT chr11-11. G-NUMT chr11-15 was detected in all 15 patients by PCR. Seven control individuals investigated from G-NUMT chr11-15 by PCR were positive for the specific amplicon. None of the G-NUMTs identified here interfered with ORF of any nuclear genes.

\section{$\underline{\text { Discussion }}$}

In 25 OSCC-GB patients investigated here, none harbour somatic NUMTs. Out of the 9 potential S-NUMT candidates identified by computational approach, none of them could be verified by PCR as somatic events. We have identified a number of germline NUMTs in oral cancer patients. But our study lacks the sample size required for estimating association of the observed G-NUMTs with oral cancer. The only G-NUMT recurrently observed in our sample set (chr-11-15) was also found to be present in blood DNA derived from non-cancer individual.

In a recent study, Ju et al., 2015 have observed S-NUMTs in 12 breast cancer samples. Among these, only 10 are primary patient samples out of a total of 559 samples harbouring various types of tumours (ALL, AML, breast cancer, lung cancer, colorectal cancer, RCC, melanoma, glioma, prostate cancer etc.) studied in the TCGA project. These events could not be correlated with any clinical phenotype of cancer and were considered as passenger events. Our result, combined with the reports previously mentioned, indicates that S-NUMTs are ambiguous in nature and probably they occur in certain specific cancers (Ju et al., 2015; Yuan et al., 2017) and not others e.g. gingivobuccal oral squamous cell carcinoma.

To conclude, we did not find any OSCC-GB specific NUMT. Whether any or all of the G-NUMTS detected by u are novel polymorphic NUMTs remain to be verified in large data set. Our study of germline NUMTs in these patients did not reveal any evidence for association with OSCCGB.

\section{Acknowledgements}

We acknowledge the ICGC India Project team and the Core Technologies Research Initiative (CoTeRI) of NIBMG for generation of the sequencing data.

\section{Declaration of interest}

The authors declare no conflict of interest.

\section{References}

- Singh KK, Choudhury AR, Tiwari HK. NUMTogenesis as a mechanism for development of cancer. Semin Cancer Biol. 2017 Dec;47:101-109. doi: 10.1016/j.semcancer.2017.05.003. Epub 2017 May 13. Review. PubMed PMID: 28511886 ; PubMed Central PMCID: PMC5683947. 
- Gellissen G, Michaelis G. Gene transfer. Mitochondria to nucleus. Ann N Y Acad Sci. 1987;503:391-401. Review. PubMed PMID: 3304081.

- Perna NT, Kocher TD. Mitochondrial DNA: molecular fossils in the nucleus. Curr Biol. 1996 Feb 1;6(2):128-9. Review. PubMed PMID: 8673455.

- Turner C, Killoran C, Thomas NS, Rosenberg M, Chuzhanova NA, Johnston J, Kemel Y, Cooper DN, Biesecker LG. Human genetic disease caused by de novo mitochondrialnuclear DNA transfer. Hum Genet. 2003 Mar;112(3):303-9. Epub 2003 Jan 25. PubMed PMID: 12545275.

- Goldin E, Stahl S, Cooney AM, Kaneski CR, Gupta S, Brady RO, Ellis JR, Schiffmann R. Transfer of a mitochondrial DNA fragment to MCOLN1 causes an inherited case of mucolipidosis IV. Hum Mutat. 2004 Dec;24(6):460-5. PubMed PMID: 15523648.

- India Project Team of the International Cancer Genome Consortium. Mutationallandscape of gingivo-buccal oral squamous cell carcinoma reveals new recurrently-mutated genes and molecular subgroups. Nat Commun. 2013;4:2873.

- Li H. (2013) Aligning sequence reads, clone sequences and assembly contigs with BWA-MEM. arXiv:1303.3997v1 [q-bio.GN]

- Dayama G, Emery SB, Kidd JM, Mills RE. The genomic landscape of polymorphic human nuclear mitochondrial insertions. Nucleic Acids Res. 2014 Nov 10;42(20):12640-9. doi: 10.1093/nar/gku1038. Epub 2014 Oct 27. PubMed PMID:25348406; PubMed Central PMCID: PMC4227756.

- Cui Y, Chen X, Luo H, Fan Z, Luo J, He S, Yue H, Zhang P, Chen R. BioCircos.js: an interactive Circos JavaScript library for biological data visualization on web applications. Bioinformatics. 2016 Jun 1;32(11):1740-2. doi:10.1093/bioinformatics/btw041. Epub 2016 Jan 27. PubMed PMID: 26819473.

- Robinson JT, Thorvaldsdóttir H, Winckler W, Guttman M, Lander ES, Getz G, Mesirov JP. Integrative genomics viewer. Nat Biotechnol. 2011 Jan;29(1):24-6.

- Ju YS, Tubio JM, Mifsud W, Fu B, Davies HR, Ramakrishna M, Li Y, Yates L, Gundem G, Tarpey PS, Behjati S, Papaemmanuil E, Martin S, Fullam A, Gerstung M; ICGC Prostate Cancer Working Group; ICGC Bone Cancer Working Group; ICGC Breast Cancer Working Group, Nangalia J, Green AR, Caldas C, Borg Å, Tutt A, Lee MT, van't Veer L, Tan BK, Aparicio S, Span PN, Martens JW, Knappskog S, Vincent-Salomon A, BørresenDale AL, Eyfjörd JE, Myklebost O, Flanagan AM, Foster C, Neal DE, Cooper C, Eeles R, Bova SG, Lakhani SR, Desmedt C, Thomas G, Richardson AL, Purdie CA, Thompson AM, McDermott U, Yang F, Nik-Zainal S, Campbell PJ, Stratton MR. Frequent somatic transfer of mitochondrial DNA into the nuclear genome of human cancer cells. Genome Res. 2015 Jun;25(6):814-24. doi: 10.1101/gr.190470.115. Epub 2015 May 11. 
Erratum in: Genome Res. 2016 May;26(5):717.2. PubMed PMID: 25963125; PubMed Central PMCID: PMC4448678.

- Yuan Yuan, Young Seok Ju, Youngwook Kim, Jun Li, Yumeng Wang, Yang Yang, Inigo Martincorena, Chad J. Creighton, John N. Weinstein, Yanxun Xu, Leng Han, Hyung-Lae Kim, Hidewaki Nakagawa, Keunchil Park, Peter J. Campbell, Han Liang, PCAWG Network. Comprehensive Molecular Characterization of Mitochondrial Genomes in Human Cancers. bioRxiv 161356; doi: https://doi.org/10.1101/161356 